\title{
Real-Time Evaluation of 26-GBaud PAM-4 Intensity Modulation and Direct Detection Systems for Data-Center Interconnects
}

Eiselt, Nicklas; Griesser, Helmut; Wei, Jinlong; Dochhan, Annika ; Eiselt, Michael; Elbers, Jörg-Peter; Vegas Olmos, Juan José; Tafur Monroy, Idelfonso

\section{Published in:}

Proceedings of Optical Fiber Communications 2016

Publication date:

2016

Document Version

Peer reviewed version

Link back to DTU Orbit

Citation (APA):

Eiselt, N., Griesser, H., Wei, J., Dochhan, A., Eiselt, M., Elbers, J-P., Vegas Olmos, J. J., \& Tafur Monroy, I. (2016). Real-Time Evaluation of 26-GBaud PAM-4 Intensity Modulation and Direct Detection Systems for DataCenter Interconnects. In Proceedings of Optical Fiber Communications 2016 [7537318] Optical Society of America (OSA).

\section{General rights}

Copyright and moral rights for the publications made accessible in the public portal are retained by the authors and/or other copyright owners and it is a condition of accessing publications that users recognise and abide by the legal requirements associated with these rights.

- Users may download and print one copy of any publication from the public portal for the purpose of private study or research.

- You may not further distribute the material or use it for any profit-making activity or commercial gain

- You may freely distribute the URL identifying the publication in the public portal 


\title{
Real-Time Evaluation of 26-GBaud PAM-4 Intensity Modulation and Direct Detection Systems for Data-Center Interconnects
}

\author{
Nicklas Eiselt $^{1,2}$, Helmut Griesser ${ }^{1}$, Jinlong Wei ${ }^{1}$, Annika Dochhan ${ }^{1}$, Michael Eiselt ${ }^{1}$, Jörg-Peter Elbers ${ }^{1}$, Juan \\ José Vegas-Olmos ${ }^{2}$, and Idelfonso Tafur Monroy ${ }^{2}$ \\ (1) ADVA Optical Networking SE, Märzenquelle 1-3, 98617 Meiningen, Germany; \\ (2) Technical University of Denmark (DTU), Department of Photonics Engineering, Ørsteds Plads, Build. 343, DK-2800. \\ niei@fotonik.dtu.dk
}

\begin{abstract}
Real-time transmission with 26-GBaud PAM-4 as a promising modulation format for data-center interconnects with operation in C-band is evaluated. For an OSNR penalty below $2 \mathrm{~dB}$ a dispersion tolerance of up to $10 \mathrm{~km}$ of SSMF is achieved.

OCIS codes: (060.2330) Fiber optics communications; (060.4080) Modulation
\end{abstract}

\section{PAM-4 for Data-center Interconnects}

The Internet traffic has seen exponential growth driven by bandwidth hungry applications such as high definition TV, online social networking, and cloud computing. In 2016, $90 \%$ of the global Internet/IP WAN traffic will pass through data centers. Higher order modulation formats are enabling an upgrade in spectral efficiency to handle the large amount of data traffic [1]. For intra-data center connections, research efforts are focused on intensity modulation and direct detection (IMDD) based short-reach fiber links (from $500 \mathrm{~m}, 2 \mathrm{~km}$ to $10 \mathrm{~km}$ ) supporting $400 \mathrm{G}$ with 8 channels of $50-\mathrm{Gb} / \mathrm{s}[2,3]$ or 4 channels of $100-\mathrm{Gb} / \mathrm{s}[4,5]$ data rates. PAM-4 and DMT are the dominant modulation formats considered for this application. Recently, the IEEE P802.3bs 400 GbE Task Force has adopted PAM-4 as an industrial standard.

For inter-data center connections, the geographic areas covered extend well beyond the optical interface standards, for example up to $100 \mathrm{~km}$ or more depending on the service supported. For such applications, optically amplified WDM systems are specified, which require placing the channels in the 1550 -nm window. One possible technical solution is scaling down from the long-haul coherent systems, which offers high margins but retains high cost together with high power consumption. On the other hand, scaling up from the short reach IMDD based client optics is a cost-effective approach but challenging in terms of OSNR performance and chromatic dispersion tolerance. Demonstrations have shown that 56-Gb/s IMDD DMT on 8 channels [6] can successfully bridge $240 \mathrm{~km}$ of SMF, and a single channel 112-Gb/s PAM-4 signal [4] transmission was demonstrated over $80 \mathrm{~km} \mathrm{SMF}$ with the aid of dispersion compensating fiber and maximum likelihood sequence estimation. However, these demonstrations utilized offline processing and involved intensive digital signal processing (DSP). 25-GBaud real-time PAM-4 has been demonstrated for transmission over $10 \mathrm{~km}$ SMF [3], but only under a high optical signal to noise ratio (OSNR) condition, since no optical amplification was involved.

Here, we demonstrate, a real-time end-to-end 52-Gb/s IMDD based PAM-4 system in the 1550-nm band with restrained OSNR conditions and simple FFE-DFE equalization. The effects of fiber dispersion and optical noise on the system performance are examined. We also identify the optimal bandwidth of optical filters.

\section{Experimental Setup}

Fig. 1 shows the experimental setup. The real-time evaluation of PAM-4 was done using a low-power, low-latency PHY circuit with retiming and equalizer functions. The PHY circuit delivered a 26-GBaud analog PAM-4 signal via a digital-to-analog converter (DAC). The SNR of the transmitted electrical eye was measured as $27 \mathrm{~dB}$. Additionally, the PHY provided a programmable pre-equalizer and a PAM-4 level shifting function (Fig. 2a) at the transmit side to compensate for the limited bandwidth and the nonlinear transfer function of the modulator. The

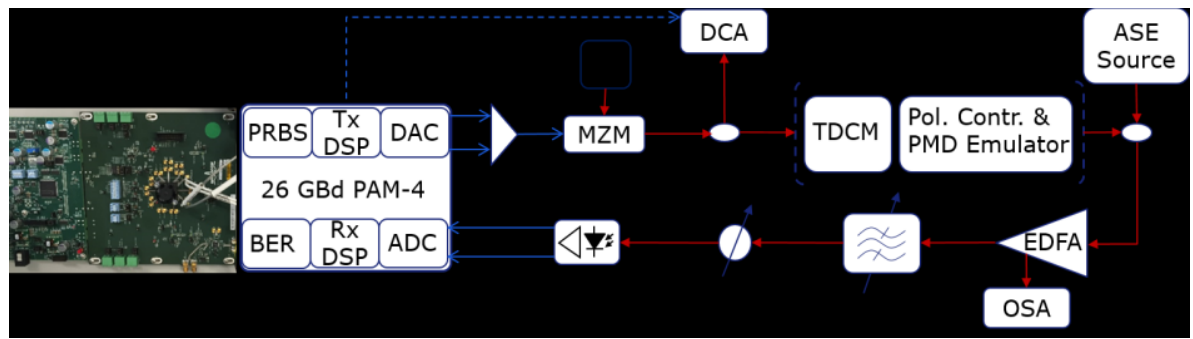

Fig. 1: Test Setup for 26 GBaud PAM-4 transmission (DCA: Digital Communication Analyzer, TDCM: Tunable Dispersion Compensator Module, VOA: Variable Optical Attenuator, MZM: Mach-Zehnder Modulator, PMD: Polarization Mode Dispersion, PIN/TIA: PIN photodiode with linear transimpedance amplifier, OSA: Optical Spectrum Analyzer, EDFA: Erbium Doped Fiber Amplifier). 
PHY receiver had an integrated ADC and an adaptive symbol-spaced FFE-DFE equalizer with adaptive thresholds prior to the PRBS bit error checker.

The differential output of the PAM-4 eval board was driving a 40G $\mathrm{LiNbO}_{3} \mathrm{MZM}$ via a linear amplifier with $35 \mathrm{GHz}$ bandwidth. An electrical attenuator after the amplifier controlled the drive amplitude into the modulator. In the optical setup, an in-line power meter (Eigenlight) after the MZM was used to determine the operating point of the MZM, while the optical eye was monitored by means of a sampling oscilloscope. Different optical effects were emulated in the setup, depending on the considered transmission scenario. Chromatic dispersion (CD) was introduced by a TDCM with an operating bandwidth of $\sim 40 \mathrm{GHz}$. Differential group delay (DGD) was introduced using a polarization controller and a PMD emulator. To ensure the worst case scenario with equal signal portions on both, the slow and the fast axis, the polarization controller was adjusted for maximum BER. To determine the OSNR requirement, noise was added from an ASE (amplified spontaneous emission) source to the signal via a 3-dB coupler. The OSNR was measured using an optical spectrum analyzer (OSA) and normalized to $0.1 \mathrm{~nm}$. A variable filter (Waveshaper) with $100 \mathrm{GHz}$ optical bandwidth, representing the DeMux in a WDM system, was used to limit the noise. For bandwidth requirement evaluation this bandwidth is changed later on. At the receiver, a linear PIN/TIA combination with $\sim 37 \mathrm{GHz}$ electrical bandwidth was used, whose electrical output was sent back to the eval board. Since most components for the experimental evaluation have much higher bandwidth than actually required, it can be considered as a "golden" setup providing a benchmark for the performance of PAM-4.

\section{Transmission Results of 26 GBaud real-time PAM-4}

The dedicated PRBS generators and checkers within the PHY circuit are used for the PAM-4 evaluation. All the following results are achieved with a prbs (pseudo random binary sequence) of length $2^{31}-1$ at an optical carrier frequency of $194.1 \mathrm{THz}$.

Fig. 2c illustrates the BER vs. OSNR performance of PAM-4 for optical back-to-back (b2b) transmission in combination with different modulator bias-points and different drive levels. The round markers display the optical b2b performance with equally spaced levels of the drive signal (Fig. 2a), and the MZM biased at the quadrature point. In order to achieve equidistant levels of the optical power, the electrical signal amplitude must be small, resulting in a low extinction ratio (approx. $4.5 \mathrm{~dB}$ ) and a large DC component. This constellation shows a very good performance for high OSNR values (BER $<10^{-9}$ ), but a large OSNR penalty at the HD-FEC limit of $10^{-3}$ BER can be observed. As shown by the squared markers, a significantly better performance can be achieved by choosing the drive levels to compensate for the nonlinear MZM transfer function (Fig. 2b). The modulator is still biased at the quadrature point, but is now driven at full $\mathrm{V}_{\pi}$ range. Thus, a high extinction ratio can be achieved. At the FEC-limit, the performance is improved by more than $5 \mathrm{~dB}$, but an error floor around a BER of $10^{-8}$ appears. A further performance improvement at the FEC limit can be achieved by changing the bias point, maintaining the same adjusted drive levels. As shown by the triangular markers in Fig. 2c, small bias changes reducing the average power can result in more than $1 \mathrm{~dB}$ OSNR improvement at the FEC-limit. Since the signal-ASE beat noise is larger at high power levels than at low power levels, a bigger upper eye opening improves the noise tolerance. However, the lower eye opening is reduced, and a higher error floor can be observed. By raising the bias-point, the lower eye is enlarged, while the upper eye gets smaller, and the noise tolerance is reduced (+ markers).

In Fig. 3a, the required OSNR (ROSNR) for a BER of $10^{-3}$ is shown vs. the chromatic dispersion for the different MZM bias settings of Fig. 2c). In contrast to optical noise, the lower eye of the PAM-4 signal suffers most from CD. As the CD increases, the impact to the lower eye becomes larger than the impact of optical noise to the upper eye, and thus we see an improved dispersion tolerance of the "raised" PAM-4 signal. At zero or low dispersion values, the effect of optical noise is dominant and hence, the "lowered" signal shows the best performance. Nevertheless,

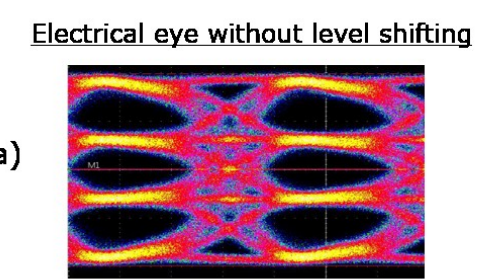

Electrical eye with level shifting

b)

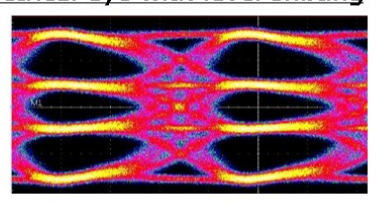

c)

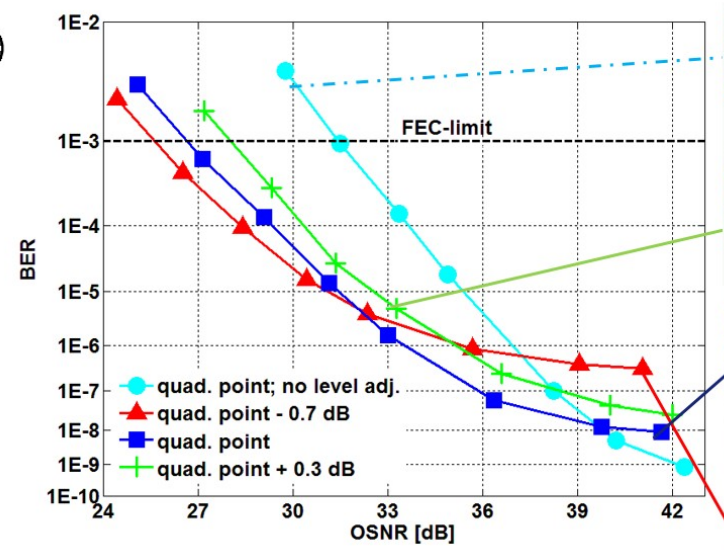

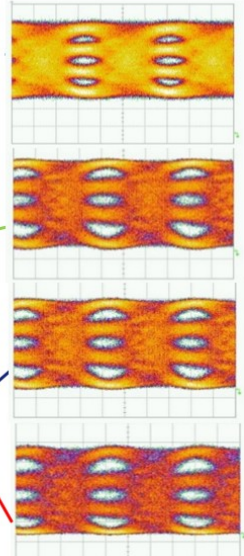

Fig. 2: a) and b) electrical output of the PHY with different PAM-4 settings, and c) optical back-to-back results. 
avoid adjustable per-channel CD compensation, the required CD tolerance is approximately $+/-180 \mathrm{ps} / \mathrm{nm}$, corresponding to $10 \mathrm{~km} \mathrm{SSMF}$. For equally spaced eyes, this CD results in a 1-dB OSNR penalty. Figure $3 \mathrm{~b}$ shows the sensitivity of PAM-4 towards DGD. For all settings, we can observe a 1-dB OSNR penalty at a DGD of approximately $15 \mathrm{ps}$. Therefore, DGD can be seen as negligible for a typical 80-km link [7]. Furthermore, the tolerance of 26-GBd PAM-4 against optical bandwidth limitations is demonstrated in Fig. 3d and 3e. Fig. 3d illustrates the ROSNR for a BER of $10^{-3}$ vs. the 3-dB filter bandwidth of the receive filter. For the 26-GBd PAM-4 signal, a 1-dB penalty can be observed at an optical bandwidth of approximately $28 \mathrm{GHz}$. In Fig. $3 \mathrm{~d}$ the optical b2b performance with a multiplexer and demultiplexer, both with an optical bandwidth of $39 \mathrm{GHz}$, is compared to the performance with a waveshaper of approximately $100 \mathrm{GHz}$ bandwidth. A small performance degradation is seen only at higher OSNR values. At the FEC limit, no performance difference is recognizable, as the noise masks the bandwidth limitation.



a)

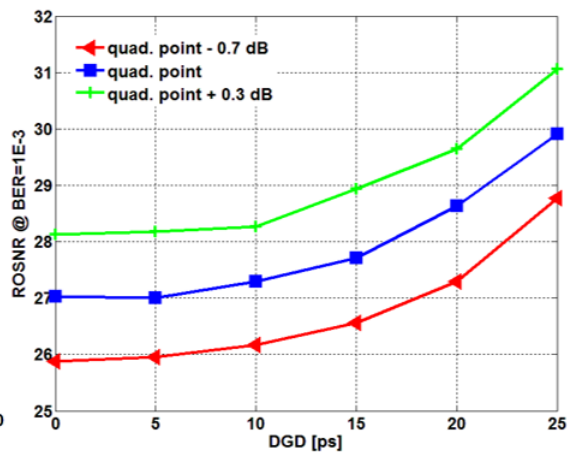

b)

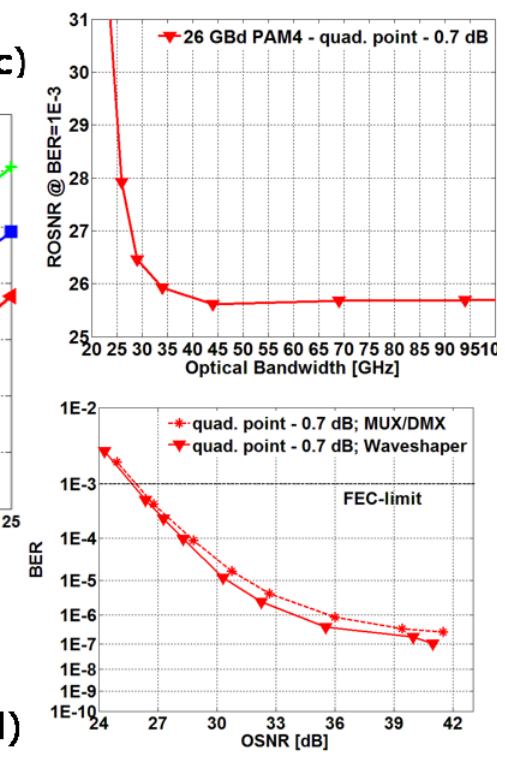

Fig. 3: a) and b) electrical output of the PHY with different PAM-4 settings, and c) optical back-to-back results.

\section{Conclusion}

On the road to $400 \mathrm{G}$ for the next generation of data center interconnects, we discussed and showed the performance of a single wavelength 26-GBd real-time PAM-4 transmission with optical noise. The experimental investigations included the impacts of CD, DGD and the optical bandwidth. While a dispersion equivalent to $10 \mathrm{~km}$ SSMF results in a 1-dB OSNR penalty, typical DGD values and filter bandwidths lead to negligible penalties. PAM-4 can therefore be seen as a promising candidate for low-cost high-rate data center interconnects.

\section{Acknowledgement}

The authors wish to thank Broadcom Inc. for the support during the experiments. The work was in part funded by the European Commission in the Marie Curie projects ABACUS and CEEOALAN and by the German ministry for education and research (BMBF) under Grant 16BP12400 as part of the SASER ADVAntage-NET project.

\section{References}

[1] J. L. Wei, et al., "400 Gigabit Ethernet using advanced modulation formats: performance, complexity, and power dissipation," IEEE Commun. Magazine,

[2] C. Chen, et al., "Transmission of 56-Gb/s PAM-4 over 26-km Single Mode Fiber Using Maximum Likelihood Sequence Estimation," OFC'15, Paper Th4A.5.

[3] M. Mazzini, et al., "25 GBaud PAM-4 error free transmission over both single mode fiber and multimode fiber in a QSFP form factor based on Silicon Photonics," Proc. OFC 2015, Paper PDP Th5b.3.

[4] D. Sadot, et al., "Single channel $112 \mathrm{~Gb} / \mathrm{s}$ PAM4 at 56Gbaud with digital signal processing for data center applications," Proc. OFC 2015, Paper Th2A.67.

[5] P. Dong et al., "Four-channel 100-Gb/s per channel Discrete Multi-Tone modulation using silicon photonic integrated circuits", Proc. OFC 2015, Paper PDP W4H.5.

[6] A. Dochhan, H. Griesser, M. Eiselt, and J.-P. Elbers, "Solutions for 80 km DWDM Systems," Proc. OFC 2015, Paper Th3A.1.

[7] M. Eiselt, B. Teipen, "Requirements for 100-Gb/s Metro Networks“, Proc. OFC 2009, Paper OTuN6. 